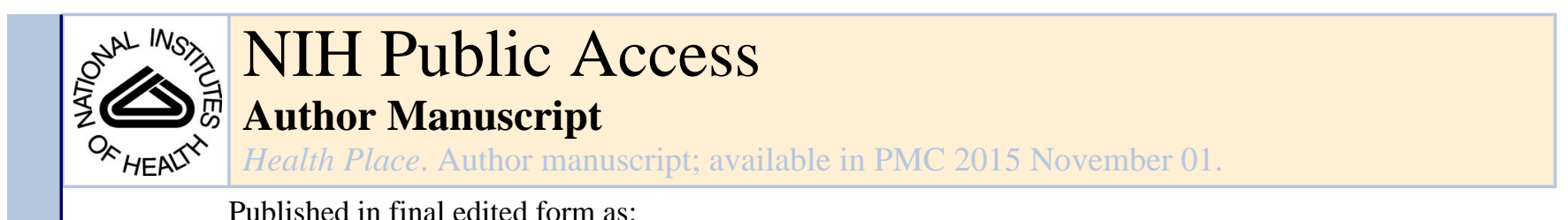

Published in final edited form as:

Health Place. 2014 November ; 0: 215-225. doi:10.1016/j.healthplace.2014.09.007.

\title{
Conceptualizing and Comparing Neighborhood and Activity Space Measures for Food Environment Research
}

\author{
Thomas W. Crawford, PhD [Banpu Endowed Chair], \\ 3694 West Pine Mall Center for Sustainability Saint Louis University St. Louis, MO 63108 \\ Stephanie B. Jilcott Pitts, PhD [Assistant Professor], \\ East Carolina University, Department of Public Health 600 Moye Blvd, MS 660, Greenville, NC \\ 27834 jilcotts@ecu.edu; Telephone: (252) 744-5572; Fax: (252) 744-4008
}

Jared T. McGuirt, MPH [Research Assistant],

University of North Carolina at Chapel Hill, Department of Nutrition Chapel Hill, NC 27599

mcguirtj@live.unc.edu Telephone: (910) 249-2296

Thomas C. Keyserling, MD, MPH [Professor], and

Department of Medicine, School of Medicine, University of North Carolina at Chapel Hill

jato@med.unc.edu; Telephone: 919-966-2276; Fax: 919-966-2274

\begin{abstract}
Alice S. Ammerman, DrPH, RD [Professor]
Department of Nutrition, Gillings School of Global Public Health, Director, Center for Health

Promotion and Disease Prevention, University of North Carolina at Chapel Hill CB\# 7426, Chapel

Hill, NC 27599-7426 alice_ammerman@unc.edu; Telephone: 919 966-6082, FAX 919 966-3374
\end{abstract}

\section{Abstract}

\begin{abstract}
Greater accessibility to geospatial technologies has led to a surge of spatialized public health research, much of which has focused on food environments. The purpose of this study was to analyze differing spatial measures of exposure to supermarkets and farmers' markets among women of reproductive age in eastern North Carolina. Exposure measures were derived using participant-defined neighborhoods, investigator-defined road network neighborhoods, and activity spaces incorporating participants' time space behaviors. Results showed that mean area for participant-defined neighborhoods ( 0.04 sq. miles) was much smaller than 2.0 mile road network neighborhoods ( 3.11 sq. miles) and activity spaces (26.36 sq. miles), and that activity spaces provided the greatest market exposure. The traditional residential neighborhood concept may not be particularly relevant for all places. Time-space approaches capturing activity space may be more relevant, particularly if integrated with mixed methods strategies.
\end{abstract}

(C) 2014 Elsevier Ltd. All rights reserved.

(Corresponding) crawfordtw@slu.edu; Telephone (314) 977-9861.

Publisher's Disclaimer: This is a PDF file of an unedited manuscript that has been accepted for publication. As a service to our customers we are providing this early version of the manuscript. The manuscript will undergo copyediting, typesetting, and review of the resulting proof before it is published in its final citable form. Please note that during the production process errors may be discovered which could affect the content, and all legal disclaimers that apply to the journal pertain. 


\section{Introduction}

For public health researchers, the concept of neighborhood is intuitively appealing, yet operationally challenging. Its intuitive appeal arguably stems from its connection to what Egenhofer and Mark (1995) refer to as "naive geography", which is a geography that captures and reflects the way people think and reason about geographic space and time, both consciously and subconsciously. The term "naive" is not meant as a pejorative descriptor but suggests an instinctive or spontaneous nature. People are likely to have immediate images and thoughts arise if they are asked to "describe your neighborhood". These instinctive, internal responses may deal with concepts or descriptors of neighborhood size, economic status, attractiveness, families or individuals, events within the year or across years, and educational, recreational, or commercial sites. Meegan and Mitchell (2001, p.2172) define neighborhood as a "key living space through which people get access to material and social resources, across which they pass to reach other opportunities and which symbolizes aspects of the identity of those living there to themselves and to outsiders."

The intuitive nature of neighborhood belies it epistemological difficulties. The title of one recent article states that measuring neighborhood is a "conundrum" (Nicotera, 2007 p.26). The variety and challenges of methods to measure neighborhood have resulted in reviews that compare and critique different methods of neighborhood measurement (Nicotera, 2007; Larson et al. 2009; DeMarco and DeMarco, 2010). Recent decades have witnessed a surge in research that empirically examines neighborhood and neighborhood effects on health behaviors and outcomes (Sampson et al., 2002; Entwisle, 2007; Matthew, 2008; Chaix et al., 2013; LeDoux and Vojnovic, 2013; James et al., 2014).

One possible reason for this surge is the relatively recent diffusion of geospatial technologies and spatial perspectives to a wider research community including the integration and analysis capabilities afforded by technologies such as geographic information systems (GIS) and global positioning systems (GPS). However, beyond simple easing of technology challenges, there are substantive theoretical reasons that have led to increased public health studies using the concept of "neighborhood". The ecological theory posits that not only do individual-level characteristics and social constructs contribute to health, but purports that spatial contexts are often important, enabling opportunities or imposing constraints for individual behaviors that are linked to health outcomes (Davison and Birch, 2001; Rayner, 2009). The process of geocoding individuals and thereby "putting people into place" (Entwisle, 2007 p. 687), when combined with other spatial data layers representing different attributes of place, enables researchers to create quantitative measures of spatial context.

The quantitative paradigm of spatialized health research has led to debate regarding the role of neighborhood effects on health. Diez Roux (2001) cautions against uncritical use of quantitative neighborhood measures for inferential analysis and argues the need to develop theory and testable hypotheses for which concepts and empirical measurements of neighborhood are justified. Important to this challenge is the need for researchers to be more precise in their definitions of neighborhood (Diez Roux, 2001). 
With respect to neighborhood research, Cummings warns against the "local trap" (Cummins, 2007 p.355). The local trap, a phrase originally used in studies of sustainable development, refers to the danger involved with uncritically assuming that local government decision making (Purcell and Brown, 2005; Purcell, 2006) and local food systems (Born and Purcell, 2006) are inherently more socially just and ecologically sustainable than larger scale processes (i.e. non-local). Cummins adapts the 'local trap' argument to warn that the neighborhood scale, as one of the most local scales beyond the individual body, is not necessarily the most meaningful or important spatial scale at which to contextualize health behaviors and outcomes. Cummins states that researchers should ask how individual exposure to 'context' can be better conceptualized in ways more meaningful than the local neighborhood. Cummins points out research using GIS to construct time-space trajectories (Kwan et al., 2003; Kwan, 2004; Kwan and Lee, 2004) and ethnographies (Matthews et al. 2005) which reveals non-local spaces beyond the neighborhood scale that individuals interact with during daily rhythms. In the present paper, we created neighborhood food exposure measures and compared them to alternative activity space measures created from time-space travel diary data. We compare neighborhood and activity space using data from a pilot study in eastern North Carolina to ask the basic questions: how do people (nonresearchers) define a neighborhood, how do researchers define neighborhood, how many healthy food venues are located in neighborhoods, and how does this differ for activity spaces?

\subsection{Measuring Neighborhood}

Chaix et al. (2009) articulate key theoretical considerations for the definition of neighborhoods. An initial distinction can be made between territorial neighborhoods versus ego-centered neighborhoods. Territorial neighborhoods are mutually exclusive and bounded areas that make up a territory. Administrative boundaries or enumeration units such as individual census tracts or census blockgroups are most commonly used to operationally define territorial neighborhoods in health research (Ford and Dzewaltowski, 2008; Leal and Chaix, 2010; DeMarco and Demarco, 2010). Ecological analyses have identified associations between aggregate measures of race and socioeconomic status and prevalence of healthy food venues (Morland et al., 2002; Morland and Filomena, 2007; Larson et al., 2008). For individual level analyses, geocoded individuals are assigned characteristics of the neighborhood (e.g. census blockgroup or tract) in which they reside, a process that is easily implemented as a point-in-polygon GIS analytical function. A limitation of this approach is that individuals in a territorial neighborhood such as a census blockgroup typically are spatially dispersed throughout the territory so that some individuals may be centrally located while others are very near the administrative boundary. Individuals located at the boundary edges may in fact interact more intensively in adjacent blockgroups just across the border.

In contrast to territorial neighborhoods, ego-centered neighborhoods are not mutually exclusive territories and instead are centered on individuals' residences so that overlap of individuals residing in close proximity is possible, what Chaix et al. (2009, p. 1306) refer to as "sliding territories" as opposed to "fixed territories". These neighborhoods define individual exposure areas and don't have fixed boundaries but instead have sliding boundaries that shift depending on the focal residence. Following Chaix et al. (2009), and 
because the present research is interested in individual or multi-level analyses as opposed to ecological analyses, the remaining discussion of neighborhood focuses solely on egocentered neighborhoods.

In terms of neighborhood scale, neighborhoods may be either individual-specific or uniform. Uniform neighborhoods enforce the same scale size for every individual - typically implemented as a uniform circular buffer distance from the residence (Spielman and Yoo, 2009). Individual-specificity recognizes that individuals may have differing perceptions of neighborhood scales depending on life course and sociodemographic characteristics (Guest and Lee, 1984). Individual-specificity is implicit with the use of resident defined neighborhoods.

Neighborhoods may either be isotropic or oriented. A circular buffer neighborhood centered on the residence exemplifies isotropic. A street network buffer neighborhood where local street configurations created oriented (i.e. anisotropic) neighborhoods of non-uniform shapes. Another distinction is between residents' perceived versus objectively experienced neighborhoods (Chaix et al., 2009) both of which exemplify resident-defined as opposed to researcher-imposed neighborhoods.

Discussion thus far has focused on residential neighborhoods as opposed to non-residential environments. Indeed, most studies focus on the local residential neighborhood, however defined. However, various scholars (Kwan and Weber, 2003; Inagami et al., 2007; Matthews, 2008; Rainham et al., 2009; Saarloos et al., 2009) as well as Cummins' (2009) 'local trap' commentary, question this focus and recognize that individuals are exposed to various environments beyond the local residential neighborhood as they engage in daily activities. Chaix refers to this as the 'residential trap' (Chaix, 2009, p.1307). Residents may perceive internally and express externally to researchers their own personal neighborhood boundaries in a biased manner that reflects neighborhood as they want them to be rather than based on an exposure area related to their actual behavior. Viewing exposure as an activity space (described below), which includes the daily/ regular experience of multiple environments, is an alternative to the sole focus on residential neighborhood that has potential to incorporate multiple sites of exposure beyond the residential neighborhood.

\subsection{Measuring Activity Space}

Activity spaces are defined by Gesler and Albert (2000) as "the local areas within which people move or travel in the course of their daily activities". As such, activity spaces can extend well beyond the neighborhood scale to capture a fuller array of exposures. Activity space has been examined by researchers across various traditions including medical geography, spatial behavior, time-space studies, transportation studies, and humanenvironment interactions. While activity space has been utilized by various health researchers (see Sherman et al., 2005; Sarloos et al., 2009; Townley et al., 2009; Mason, 2010; Rainham et al., 2010; Villanueva et al., 2012), there are few studies for the food environment that focus on activity space. Two notable examples are Zenk et al. (2011) and Kestens et al. (2010). Zenk et al. (2011) used GPS tracking applied to a sample of 120 participants in Detroit, Michigan over seven days to measure activity spaces and relate them to dietary and physical behaviors. Zenk et al. (2011) found that activity spaces were larger 
than residential neighborhoods, that no residential neighborhoods contained a single supermarket, and that statistically significant relationships between activity space environmental features and dietary behavior. Kestens et al. (2010) combined travel survey data with food store locations in Montreal and Quebec City, Canada for a sample of approximately 200,000 adults to create measures of foodscape exposures that differed from traditional residential-based measures. Both studies conclude that activity space approaches show promise to provide new insights regarding environmental influences on diet and obesity-related behaviors.

Similar to neighborhoods, there are multiple ways to conceptualize and measure activity space that are reviewed in Sherman et al. (2005). The most common approach is the standard deviational ellipse (SDE) applying either a one or two standard deviation threshold. With the advent of GPS tracking, an alternative to the SDE is to capture a participant's traveled route throughout the study period and buffer the linear route by a defined distance such as 0.5 or 1.0 miles. Unlike the SDE, this second approach trims the resulting activity space to conform more faithfully to the actual spaces of the individual's trajectory.

The Kestens et al. (2010) study differed from both approaches by using kernel density methods applied to food store point data to create foodscape exposure surfaces (see also Goldsberry et al. 2010) and overlaid destination point data to retrieve the surface density values as individual level foodscape exposure measures.

\subsection{Research Questions}

Having reviewed the literature on neighborhoods and activity spaces, we now refine our basic motivating question as indicated in the article title to specify three more formal research questions:

1. How do areal sizes of self-defined neighborhoods differ from network distance neighborhoods and activity space areas?

2. How do measures of exposure to healthy food venues (i.e. supermarkets and farmers' markets) differ depending on the use of self-defined neighborhoods, road network neighborhoods and activity spaces?

3. How do measures of exposure to healthy food venues differ by urban, employment, and racial status depending on the use of self-defined neighborhoods, road network neighborhoods and activity spaces?

Answers to these questions are important to both the research and policy arenas. As stated earlier, much work has focused on the neighborhood as a potential mediating context for a variety of health related behaviors. In light of critiques of the neighborhood concept such as the 'local trap', if the concept of neighborhood is vindicated, then policies targeting neighborhoods as intervention sites can have greater confidence. Alternatively, answers may call into question the role of neighborhood and suggest other spatial contextual influences such as activity space.

Our focus on low income women of reproductive age is important because women of reproductive age are a critical group to study as they have influence over their family 
members' eating habits (Wild et al., 1994; Scaglioni et al., 2008). Such women also have a variety of travel patterns and activity spaces. For instance, some women might be younger, working mothers who drop children at childcare or school before heading to work, and may make a series of trip chains on the way home from work. Others may be older or unemployed and have no children, and have a smaller activity space, mainly staying in close proximity to the residential address. Still other women may be rural dwellers and have a longer distance to drive to shop for food compared to more urban women (Hillier et al., 2011; Jilcott Pitts et al., 2013). It is important to study travel patterns of a variety of demographic groups (e.g., employment status, urban or rural residence) in order to inform future interventions to promote health among various groups.

\section{Data and Methods}

\subsection{Study Setting and Participants}

This study was set in Pitt County, eastern North Carolina (2010 population estimate = 168,148). Pitt County is a U.S. Census defined metropolitan statistical area containing the incorporated municipality of Greenville, which is surrounding by largely suburban neighborhoods and rural countryside. According to the USDA's Rural-Urban Continuum classification scheme, Pitt County is coded as a type " 3 - counties in metro areas of fewer than 250,000 population" (USDA, 2013a). According to 2010 U.S. Census data there are 351 United States counties of this type containing a population of $30,738,488$. Pitt County includes a large regional hospital and a publicly funded university. Data from the U.S. Food Environment Atlas estimate that $35.9 \%$ of Pitt County adults are obese (USDA, 2013b). U.S. Census American Community Survey data from 2007-2011 estimate a Pitt County poverty rate of $24.0 \%$ which is substantially higher than the statewide rate of $16.1 \%$ and national rate of $14.3 \%$ (US Census, 2010).

The current study was ancillary to a larger Centers for Disease Control and Preventionfunded study, called "InShape" (Integrated Screening and Health Assessment, Prevention and Evaluation), designed to examine and address risk factors for heart disease among women of reproductive age $(18-44$ years). Family planning patients were eligible for InShape if they were female, age 18-44 years, English speaking, attending an initial or annual family planning visit, and had valid physical address information. Family planning clinic patients were screened for InShape eligibility, and eligible women expressing interest in participating in this ancillary qualitative study were recruited by a research assistant in the waiting area. We initially recruited 37 female participants (18-44 years). Women electing to participate were read a consent form and signed informed consent. The InShape study was approved by the Institutional Review Board of the University of North Carolina in Chapel Hill and the qualitative ancillary study was approved by the East Carolina University Institutional Review Board.

In the waiting area, women completed an InShape enrollment form, which included questions related to access to food venues, shopping patterns, and demographic variables. Upon completion of the InShape enrollment form, a question assessed whether women were willing to meet at a later scheduled date for an in-depth qualitative interview. Interested participants were contacted by phone, and interviews were scheduled in a public setting such 
as a local coffee shop or the public library. The qualitative interview included a three-day retrospective daily travel $\log$, a request for participants to hand-draw the boundary of their individual neighborhoods, and additional open-ended questions regarding food purchasing behavior. Interviews lasted approximately one hour, and participants were provided a $\$ 20$ incentive upon completion of the interview.

\subsection{Neighborhood Methods}

Neighborhood was measured using two different approaches: (1) self-defined, and (2) network distance. Prior to the scheduled interviews, all women had stated their home residence street addresses on the enrollment form. Street addresses were geocoded to a point $(\mathrm{x}, \mathrm{y})$ location using ArcGIS software and BatchGeo, a free online geocoding web application (Jilcott et al., 2011b; batchgeo, 2013). All resulting points were carefully evaluated for accuracy by comparing results with two external sources: (a) a Pitt County property parcel GIS layer, (b) Google Maps. All street addresses were confirmed to be accurately located.

2.2.1. Participant-defined neighborhood-Prior to the scheduled interviews, a series of six customized maps specific to each individual was created (Fig. 1). All maps were produced in Google Maps and printed out in hardcopy to be shown to the participant. Each map was centered directly over the participant's residence. The first map employed a large cartographic scale so that the image was zoomed in and showed greatest detail. The successive five maps were identical except that each succession employed a smaller scale by zooming out one "click" within the Google Maps interface. Map scales ranged from 1:2,000 to $1: 64,000$.

Participants were presented all six maps and were asked to mark on a single selected map their neighborhood boundary as a closed polygon using a black marker. No definition of "neighborhood" was provided by the interviewer. Participants were simply asked to "draw your neighborhood" on the single map they perceived to be most appropriate in performing this task.

The marked map was subsequently scanned and converted to a tiff format image file. The map image was georeferenced using ArcGIS software. Four registration points located at street intersections clearly evident on both the scanned image and a georeferenced air photo image were used to perform image registration using a first order affine transformation. Average root mean square error for all georeferenced scan images was less than 5 meters. Neighborhood polygon boundaries were then digitized by manual tracing using the computer mouse and tagged with a participant ID value to enable linkage with other tabular data (Fig. 2).

2.2.2. Investigator-defined Network distance neighborhood-Neighborhoods were also defined according to road network distance. Such definition requires implementing an a priori distance threshold. Prior research has used different distance thresholds, although a 1-mile distance is fairly common. We opted to use the following series of distance ranges: $0.50,1.00,1.50$, and 2.00 miles. Thus, for each participant we created four network distance neighborhoods (Fig. 3) using the Network Analyst extension 
of ArcGIS software. Together with the self-defined neighborhood, this yielded five different representations of "neighborhood" for each individual.

\subsection{Activity space methods}

Activity spaces differ conceptually from local neighborhoods in that they are based on the actual travel behavior of the individual. For each participant, activity spaces were created to represent a polygon area indicating the region in which they move throughout the landscape during their regular routines.

2.3.1 Travel Diary-During the interview and following the neighborhood mapping, participants were administered a standard travel diary instrument. We used the TravelTrac instrument which has been used successfully in other research (Jilcott et al., 2011a). Participants were guided through a memory recall of all the places that they traveled to during the prior three days. Detailed information regarding both time and location were elicited and recorded by the interviewer on the paper copy of the travel diary and on a digital audio recorder. Time spent at each location and time spent traveling to successive locations was recorded for the entire 72 hour period with as much locational detail as possible. Where possible, specific street addresses were obtained. In many cases, a specific place name and identifiable location were obtained. For example, "the McDonald's on $10^{\text {th }}$ Street". In other cases, a nearest street intersection was obtained. Each recorded entry from the travel diary was entered as a record in a tabular database. Each visited place was geocoded to a point location using a combination of county parcel data and Google Maps. Temporal and other attribute information for each journey and each place visited (including the duration of the visit) were linked to each point in the 72 hour sequence to create a time-space activity path for each individual (Fig. 4).

2.3.2. Time-weighted standard deviational ellipse-Each individual's activity space was estimated and represented spatially by creating a time-weighted standard deviational ellipse. We used standard deviational ellipses because our travel diary data did not contain linear route information that one might obtain using GPS. Linear features would enable the alternative of buffering out from these features to create activity spaces; however the point nature of our data made this approach unfeasible Our data were points containing time information regarding daily activities. For all places visited (including home residence) during the 72 hour period, locational coordinates and duration in hours at each location were retrieved from the travel diary and linked GIS database. Each point was weighted by its duration hours, and ellipses were generated according to standard formulas as implemented in ArcGIS software (Mitchell, 2005). Ellipses were generated using the one standard deviation distance which, due to the time weighted nature, theoretically should contain approximately $68 \%$ of location hours during the 72 hour period. Figure 4 visualizes two participant activity spaces in a manner similar to Hagerstrand's (1970) time-space prism and Kwan's time-space aquarium (Kwan and Lee, 2004) where the ellipse defining the activity space is extended vertically with the vertical dimension representing time. Individual activity sites are connected sequentially to reveal time-space activity paths. Figure 4 depicts paths for a 24 hour period, although the ellipses were based on the full 72 hour period. Other activity space methods exist including kernel density estimation, convex hull, and buffered 
activity locations. Standard deviational ellipses (SDE) were used here due to their common use in the literature and because with time weighted they allow one to introduce the temporal domain, something the convex hulls and buffered activity locations do not. A criticism of SDE is that its space-filling elliptical nature may bias its areal size to be larger than sizes for some other methods, although it is not clear in principal if this is necessarily the case. For example, convex hull may be larger or smaller than SDEs depending on the configuration of activity sites. Similarly, kernel density surfaces may be larger or smaller depending on parameters such as bandwidth and the density threshold applied to define the activity space. Buffered activity locations will most likely be smaller in area than SDEs but the exclude the interstitial areas between activity sites through which individuals traverse and are potentially exposed to food venues.

\subsection{Derived Variables}

Our goal was to compare participant exposure to healthy food venues according to differently defined exposure areas. There were two main types of exposure areas neighborhood and activity space. For neighborhood, there were five different neighborhood definitions - one participant-defined and four network distance. There was one activity space definition. Size in square miles was calculated for all exposure areas. Comparing differences in magnitudes of the network neighborhoods that vary incrementally by road distance thresholds was included to evaluate which distance threshold yields region sizes most similar in magnitude to the participant-defined neighborhood (i.e. perceived neighborhood) and activity space.

To quantify the cartographic scale at which participants most readily perceived and identified their neighborhoods, we recorded the scale of the map they selected for this task from the six maps they were presented with during the interview.

Supermarkets and farmers' markets were considered to be healthy food venues where participants would have the potential to purchase food that promotes positive health. Exposure measures for supermarkets and farmers' markets were calculated as the counts of the number of markets located within each defined neighborhood and activity space. GIS point-in-polygon functions were used to obtain these counts.

Supermarkets were identified from the ReferenceUSA business listings filtered according to NAICS industry classification code 445110. Downloaded listings included latitude/longitude coordinates from which point features were created. Spatial accuracy was verified through consulting corporate websites of respective supermarket brands and visual inspection using Google Maps and aerial photographs within a GIS environment. In a few selected instances, points that were visually offset from their proper location required editing to manually shift points to correct locations. Local farmers' markets were identified from the NC Farm Fresh website (http://www.ncfarmfresh.com), a state sponsored clearinghouse of market information that includes street address information. Additional ground-truthing and field staff verification supplemented information obtained from this website (McGuirt et al., 2011). Farmers' markets were geocoded as point features using a combination of Google Maps, county parcel data, and air photos. 
Basic demographic data included information on age, race, employment, and urban/rural status. Age and race data were obtained from chart review. Participants reported their employment status as either employed or unemployed. Urban residents were defined as those with a Greenville or Winterville zip code. All other Pitt County zip codes were defined as rural.

To compare areal size and supermarket and farmers' market exposure among the six exposure area types we applied the Friedman test (Siegel and Castellan, 1988), which is a non-parametric alternative to the one-way ANOVA with repeated measures. Friedman test results indicate if there is at least one significant difference between measures corresponding to the multiple exposure area types. We also performed pairwise comparisons to identify specific pairings with statistically significant differences. We applied Mann Whitney tests, a non-parametric alternative to Student's t-test, to evaluate differences between urban/rural, employed/unemployed, and black/white participants.

\section{Results}

We interviewed a total of 37 women of which 36 had complete residence address and travel diary data enabling construction of neighborhoods and activity spaces. Of these 36,34 had complete demographic data. Of these 34, 15 (44\%) reported no annual income. For the remaining 19 (56\%), the reported annual income ranged from $\$ 2,600$ to $\$ 31,772$ with a mean of $\$ 12,751$. Other descriptors for the 34 include: 53\% urban / $47 \%$ rural; 59\% black / $41 \%$ white; and $50 \%$ employed / $50 \%$ unemployed.

When presented with six maps of varying scale used to draw neighborhoods, the 1:4,000 scale map had the highest frequency of selection followed closely by the 1:8,000 scale map (Table 1). Together these two maps scales accounted for approximately $80 \%$ of the observations.

Size of exposure areas varied significantly by type of exposure area (Table 2). Participantdefined neighborhoods were the smallest in area with a median size of 0.02 square miles. The successive road network neighborhoods were increasingly larger with increasing distance thresholds, as would be expected. Activity spaces had a median area of 8.72 square miles which was notably larger than medians for all of the neighborhood types. The high mean activity space area (26.26 square miles) compared to the median reflects the fact that activity space size is not normally distributed. This was due to one outlier participant. Both means and median are included to present the fullest information, however the nonparametric nature of the Friedman test and associated pair-wise comparisons is robust to the presence of an outlier. To ease presentation of multiple comparisons, we excluded the 0.5 and 1.5 mile road network neighborhoods (Table 3). Applying a significance level of $\mathrm{p} \leq$ 0.05 throughout, all pairwise comparisons showed statistically significant differences in area sizes with the exception of the paired 2.0 mile network neighborhoods and activity spaces.

To ease presentation, the exposure variables are grouped into the categories $0,1,2$, or $3+$ markets per exposure area and relative frequencies are presented as percentages (Table 4). All self-defined neighborhoods had zero supermarket and farmers' market exposure, 
meaning that no markets were located in any of the 36 self-defined neighborhoods. For road network neighborhoods, percentages with at least one market increased with the increasing distance thresholds from 0.5 to 2.0 miles.

Pairwise comparison results (Table 5) show that supermarket exposure for self-defined neighborhoods was not significantly different from the 1.0 mile neighborhoods but had significantly lower exposure than both the 2.0 mile neighborhoods and activity spaces. Activity spaces had greater supermarket and farmers' market exposure than all neighborhood types except the 2.0 mile neighborhood for which there was no statistically significant difference.

Chi-square tests (not shown) indicated no associations between selected map scale and urban/rural residence, race, and employment statuses. Mann-Whitney test results revealed statistically significant differences for other measures (Table 5). Compared to rural participants, urban participants had larger 2.0 mile network neighborhoods and greater supermarket and farmers' market exposures. Rural participants had larger activity spaces than urban participants. Employed participants had larger self-defined neighborhoods and greater farmers' market exposure. For race (black vs. white), Mann-Whitney tests (not shown) revealed no differences in any of the measures.

\section{Discussion and Conclusion}

Within the context of the 'local trap' debate (Cummins 2007), the motivation for this research was to ask with regards to food environment research, "What's in a neighborhood?" In terms of size, we found that participants perceived their neighborhoods as being relatively small (mean $=0.04$, median $=0.02$ square miles), much smaller than census tracts or blockgroups that are frequently used to represent neighborhood. For example, our 36 participants' home residences were located in 19 tracts ranging in size from 0.72 to 82.2 square miles (mean $=18.67$, median $=5.55)$ and 28 blockgroups ranging from 0.12 to 32.66 square miles $($ mean $=6.23$, median $=0.64)$. Participant-defined neighborhoods were also smaller than all road network neighborhoods with distance thresholds from 0.5 to 2.0 miles. The 0.5 mile neighborhood, having the smallest distance threshold, necessarily had the smallest mean area of the network neighborhoods, but pairwise comparison showed even this smallest of the network neighborhoods investigated to be significantly larger than selfdefined neighborhoods. For this study, the threshold at which there would be no significant difference from self-defined neighborhoods would clearly be less than 0.5 miles. This suggests that thresholds smaller than 0.5 miles may be warranted for researchers desiring representation of residential neighborhoods.

However, simply focusing on areal magnitude is but one dimension of neighborhood. It is possible for two differently defined neighborhood areas for an individual residence to have identical areal sizes yet cover almost completely different areas. Interestingly, we found no size differences in participant-defined neighborhoods for rural versus urban participants, although urban participants had larger 2.0 mile network neighborhoods. This is due to the denser road network of the urban fabric that leads to more roads and hence more land areas being defined into the network neighborhood with greater distance thresholds (see Oliver, 
2007). The larger participant-defined neighborhood size for employed participants suggests the importance of active employment on environmental perception compared to unemployed individuals who may be more likely to be homebound and hence have smaller perceived neighborhood areas.

Beyond the matter of neighborhood size, researchers interested in contextual effects are interested in quantities and qualities of food venues within neighborhoods. Strikingly for our sample of 36 low income women, not a single supermarket or farmers' market was located inside any of the 36 self-defined neighborhoods, a finding in agreement with Zenk et al. (2011).

There are perhaps three ways to interpret the result of no markets being located in the selfdefined neighborhoods. It is possible that the notion of perceived neighbourhood is not very relevant for food accessibility. People may have a very small representation of their residential neighbourhood while having a relatively high perception of accessibility to food sources around (outside) their neighbourhood. Are we sure that the "perceived neighbourhood' is the most relevant unit of observation when considering accessibility? In other words, if one asks 'do you have good accessibility to food sources in your neighbourhood?' the answer may be 'no', but if one asks 'do you have good accessibility to food sources from home or near your neighborhood' the answer may be either 'yes' or 'no' depending on how distant food venues are from the home or neighborhood. Unlike perceived neighborhoods, the fact that activity spaces contain food venues is explained by the simple fact that the vast majority of people must travel to venues in order to obtain food, however it still is possible that these food venue sites are distant and with low spatial accessibility within the context of other activity sites that are part of the activity space.

Second and in contrast to our first interpretation, due to our sample of low income women in Pitt County, it may be that these women are more likely to live in food desert locales, and that neighborhoods for higher socio-economic women would have non-zero supermarket and farmers' market exposures. In other words, the concept of self-defined neighborhood may in fact matter across a larger sample size that is more richly stratified both socioeconomically and geographically. Place differences matter also. Rundle et al. (2009) found an inverse relationship between BMI and neighborhood healthy food density for a sample of 13,102 adults in New York City using a 0.5 mile circular residential buffer neighborhood. They also found that $63.2 \%$ of the neighborhoods had at least one supermarket, (Rundle 2009) a much higher rate than for our small sample that used a 0.5 network neighborhood for which only $5.6 \%$ had at least one supermarket. Neighborhood parameters for large metro areas like New York may not be relevant for the many individuals who do not reside in such densely populated areas. We note that within the U.S., a strong majority of population lives in metropolitan counties, and that Pitt County is a relatively small metro county. However, according to 2010 census data, a sizable $31 \%$ of the U.S. population lives in counties with smaller populations than Pitt County.

Third and returning to the notion of naive geography, it may be the case that there is a disconnect between layperson and researcher understandings of the term "neighborhood". Laypersons, as research participants, will respond to the concept of neighborhood drawing 
from their instinctive naive geography understandings. Their notion of neighborhood is more likely to be residentially focused and may even be biased to largely exclude commercial or other forms of non-residential land use that may be in close proximity to home residences, although this is a question for future research. For researchers, "neighborhood" may be simple shorthand for nearness or proximity, where they are not thinking in naive geography terms of residential neighborhood, but are thinking in more abstract spatial analytic terms related to multi-level models and spatial statistical effects and relationships. Alternatively, other researchers may be responding with the same naive geography understanding as laypersons when confronting the concept of neighborhood in their research. We speculate that in addition to a possible disconnect between researcher and research subject, there may also be a disconnect between researchers and other researchers. We suggest that researchers would benefit by future dialog at professional meetings or in professional literature regarding disciplinary and inter-disciplinary understandings of the neighborhood concept.

Our results contribute to the literature evaluating relationships between perceived neighbourhoods, activity spaces, and various health outcomes. Among seniors in rural Texas, researchers examined the association between produce consumption and perceived and objectively-measured access to fruits and vegetables, finding that lower fruit and vegetable intakes were associated with greater distance to a supermarket (Sharkey et al., 2010). In another study among low-income public housing residents in Boston, perceived, but not objectively-measured access to supermarkets was associated with greater produce intake (Caspi et al., 2012). Finally, among Kentucky adults, shopping at a farmers' market was associated with greater fruit and vegetable intake, while mere venue availability within the activity space was not associated with produce intake (Gustafson et al., 2013). Our results contribute to the literature by complicating notions of accessibility and exposure suggesting that the spatial method used might influence interpretation of accessibility/ exposure results and have potential to influence interpretation of results for actual food purchase or intake behavior, although we did not explicitly investigate these latter issues.

Our results also contribute to discourse related to the task of addressing "how can individual exposure to 'context' itself be better conceptualized?' (Cummins, 2007, p.355). In interpreting results for self-defined neighborhoods, we have indicated some generalizable complications involved with ego-centric neighborhood definitions. Our results also showed that road network neighborhoods are larger than self-defined neighborhoods even at our smallest distance threshold of 0.5 miles. Compared to self-defined neighborhoods, greater exposure to supermarkets and farmers' markets were present only with a 2.0 mile threshold. There was no statistically significant difference for 1.0 mile and self-defined neighborhoods. Results also showed substantial variation in exposure for the 2.0 mile neighborhood. This might suggest that, at least for our study area, adopting the 2.0 mile neighborhood is the preferred choice and that, in principle, similar analyses for different places could reveal appropriate place-specific distance thresholds. Variation is interpreted here as a positive because with variation there is potential for analysis to explain this variation. With no variation, there is nothing to explain in a statistical sense. Yet one must take care that variation revealed by a certain method is logically relevant in a real-world behavioral sense and is not simply expedient for subsequent statistical methodologies. It is important to also 
recognize that the road network neighborhood and exposure measures derived from it are normative measures and do not necessarily reflect actual exposure of individuals reflected in their time-space behaviors as they live, interact socially, and move about in place. Road network neighborhoods, regardless of distance thresholds, can be criticized for being investigator-defined and normative measures that are based solely on distances and geometries of road infrastructures. Speaking more generally of neighborhoods defined by secondary source boundaries, Weiss et al. (2007, p.S155) makes the observation that this approach "may be inefficient for studies involving primary data collection, as it may be impossible to discern, prior to the start of data collection, if factors of interest are present in the selected geographic areas."

Activity space offers a more promising alternative for food environment research due to the fact that it is not normative and is an objective measure of individual time-space behavior. However, it is not without difficulties. Challenges include the much greater costs of primary data collection, particularly for larger sample sizes required for robust statistical modeling. Assuming that requisite time-space behavioral data are collected, methodological choices must be made regarding how best to represent activity spaces. Our work used a timeweighted one standard deviational ellipse (SDE) due to the nature of our data which contained point location and duration information but no information on actual routes traveled. Primary data collected from GPS units allows for other more nuanced forms of activity spaces. For example, blocks adjacent to or a defined buffer zone around traveled routes could reveal more detailed representations of activity spaces as pathways of food environment exposure. Even with the advances of GPS, there still remain substantial challenges regarding protocols for data cleaning and analysis (Rainham et al. 2010).

A limitation of this research is that it was a small pilot study with limited generalizability to other places. Additionally, our study focused only on two types of food venues, so that it is possible that other venues such as fast food restaurants or convenience stores were within some of the neighborhoods. The travel diary data contained point location and duration information, but no information on actual routes traveled. We used standard deviational ellipses to conceptualize activity space, but ellipses themselves can be criticized for being abstract spatial statistical summaries. Much of the area inside SDEs is not actually visited by individuals. Another potential limitation is that we used raw exposure to healthy food venues. A relative measure, such as the Retail Food Environment Index (Babey and Diamont 2008), might be a more relevant way to assess exposure to the food environment as it portrays "relative" exposure to both healthy and less healthy food venues.

Our case study does not provide a definitive answer to the question "What's in a neighborhood?" posed by our title. Being based on a small pilot study, we are under no illusion that our analysis will provide definitive answers applicable to all places, but we believe that it can move the discourse incrementally forward by identifying some of the challenges and possible solutions related to measuring spatial exposure for food environment research. In conclusion, the 'local trap' causes food environment researchers to think critically about processes responsible for health behaviors, how they might operate at local, distant, or multiple scales; and how we should operationally measure and observe these processes. Geo-ethnography work by Matthews et al. (2005) and "relational" 
approaches argued by Cummins et al. (2007) both contextualize "people in place" but do so in ways with richer nuance that recognize and attempt to interpret complexities of timespace behaviors allowing for both quantitative measure of spatial geometries and exposures as well as social and power relations and structures. Food environment research would likely benefit by investigating mixed-methods strategies such as geo-ethnographies or relational approaches towards understanding and representing 'place'. Such strategies will likely lead to new understandings of how and where neighborhoods and activity spaces matter.

\section{Acknowledgments}

This research was supported by resources of East Carolina University's Center for Geographic Information Science.

Funding Disclosure: Research reported in this publication was supported by Cooperative Agreement Number 5U48DP001944 from the Centers for Disease Control and Prevention (CDC) and by the National Institutes of Health/ National Cancer Institute (NIH/NCI) under award number 5R03CA155362-02. The findings and conclusions in this report are those of the authors and do not necessarily represent the official position of the CDC or NIH/NCI.

\section{References}

batchgeo. [Mar., 17, 2013] batchgeo. 2013. at: http://batchgeo.com/

Babey, S.; Diamont, A.; Holbert, T. Designed for Disease: The Link Between Local Food Environments and Obesity and Diabetes. California Center for Public Health Advocacy, Policy Link; UCLA Center for Health Policy Research; Davis, CA: Los Angeles, CA: 2008.

Born B, Purcell M. Avoiding the Local Trap: scale and food systems in planning research. Journal of Planning Education and Research. 2006; 26:195-207.

Caspi C, Kawachi I, Subramanian S, Adamkiewicz G, Sorensen G. The relationship between diet and perceived and objective access to supermarkets among low-income housing residents. Social Science \& Medicine. 2012; 75(7):1254-1262. [PubMed: 22727742]

Chaix B, Merlo J, Evans D, Leal C, Havard S. Neighborhoods in eco-epidemiologic research: delimiting personal exposure areas. A response to Riva, Gauvin, Apparico, and Brodeur. Social Science \& Medicine. 2009; 69:1306-1310. [PubMed: 19692161]

Chaix B, Meline J, Duncan S, Merrien C, Karusisi N, Perchoux C, Lewin A, Labadi K, Kestens Y. GPS tracking in neighborhood and health studies: a step forward for environmental exposure assessment, a step backward for causal inference? Health \& Place. 2013; 21:46-51. [PubMed: 23425661]

Cummins S. Commentary: investigating neighborhood effects on health - avoiding the 'Local Trap'. International Journal of Epidemiology. 2007; 36:355-357. [PubMed: 17376797]

Davison K, Birch L. Childhood overweight: a contextual model and recommendations for future research. Obesity Review. 2001; 2(3):158-171.

DeMarco A, DeMarco M. Conceptualization and measurement of the neighborhood in rural settings: a systematic review of the literature. Journal of Community Psychology. 2010; 38(1):99-114.

Diez Roux A. Investigating neighborhood and area effects on health. American Journal of Public Health. 2001; 91(11):1783-1789. [PubMed: 11684601]

Egenhofer, M.; Mark, D. Naive geography.. In: Frank, A.; Kuhn, W., editors. Spatial Information Theory - a Theoretical Basis for GIS, International Conference COSIT '95, Lecture Notes in Computer Science 988. Springer-Verlag; Berlin: 1995. p. 1015

Entwisle B. Putting people into place. Demography. 2007; 44(4):687-703. [PubMed: 18232206]

Ford P, Dzewaltowski D. Disparities in obesity prevalence due to variation in the retail food environment: three testable hypotheses. Nutrition Reviews. 2008; 66(4):216-228. [PubMed: 18366535] 
Gesler, W.; Albert, D. How spatial analysis can be used in medical geography.. In: Albert, D.; Gesler, W.; Levergood, B., editors. Spatial Analysis, GIS and Remote Sensing Applications in the Health Sciences. Ann Arbor Press; Chelsea, MI: p. 11-38.

Goldsberry K, Duvall C, Howard P, Stevens J. Visualizing nutritional terrain: a geospatial analysis of pedestrian produce accessibility in Lansing, Michigan USA. Geocarto International. 2010; 25(6): 485-499.

Guest A, Lee B. How urbanites define their neighborhoods. Population and Environment. 1984; 7(1): $32-56$.

Gustafson A, Christian J, Lewis S, Moore K, Jilcott S. Food venue choice, consumer food environment, but not food venue availability with daily travel patterns are associated with dietary intake among adults, Lexington Kentucky 2011. Nutrition Journal. 2013; 12:17. [PubMed: 23360547]

Hagerstrand T. What about people in regional science? Papers in Regional Science. 1970; 24:6-21.

Hillier A, Cannuscio C, Karpyn A, McLaughlin J, Chilton M, Glanz K. How far do low-income parents travel to shop for food? Empirical evidence from two urban neighborhoods. Urban Geography. 2011; 32(5):712-729.

Inagami S, Cohen D, Finch K. Non-residential neighborhood exposures suppress neighborhood effects on self-rated health. Social Science \& Medicine. 2007; 65(8):1779-1791. [PubMed: 17614175]

James P, Berrigan D, Hart JE, Hipp JA, Hoehner CM, Kerr J, Major JM, Oka M, Laden F. Effects of buffer size and shape on associations between the built environment and energy balance. Health \& Place. 2014; 27:162-170. [PubMed: 24607875]

Jilcott S, Moore J, Wall-Bassett E, Liu H, Saelens B. Association between travel times and distances, perceived stress, food procurement practices and body mass index among female Supplemental Nutrition Assistance Program (SNAP) participants in eastern North Carolina. Journal of Nutrition Education and Behavior. 2011a; 43(5):385-389. [PubMed: 21906550]

Jilcott S, Wade S, McGuirt J, Wu Q, Lazorick S, Moore J. The association between food environment and weight status among eastern North Carolina youth. Public Health Nutrition. 2011b; 14(9): 1610-1617. [PubMed: 21486525]

Jilcott Pitts S, Wu Q, McGuirt J, Crawford T, Keyserling T, Ammerman A. Associations between access to farmers' markets and supermarkets, shopping patterns, fruit and vegetable consumption and health indicators among women of reproductive age in eastern North Carolina, USA. Public Health Nutrition. 2013; 16(11):1944-1952. [PubMed: 23701901]

Kestens Y, Lebel A, Daniel M, Theriault M, Pampalon R. Using experienced activity spaces to measure foodscape exposure. Health \& Place. 16:1094-1103. [PubMed: 20667762]

Kwan M. GIS methods in time-geographic research: geocomputation and geovisualization of human activity patterns. Geografiska Annaler B. 2004; 86:267-280.

Kwan M, Murray A, O'Kelly M, Tielfelsdorf M. Recent advances in accessibility research: representation, methodology and applications. Journal of Geographical Systems. 2003; 5:129-138.

Kwan M, Weber J. Individual accessibility revisited: implications for geographical analysis in the twenty-first century. Geographical Analysis. 2003; 35(4):341-354.

Kwan, M.; Lee, J. Geovisualization of human activity patterns using 3-D GIS: a time geographic approach.. In: Goodchild, M.; Janelle, D., editors. Spatially Integrated Social Science. Oxford University Press; New York: 2004.

Larson N, Story M, Nelson M. Neighborhood environments: disparities in access to healthy foods in the U.S. American Journal of Preventive Medicine. 2009; 36(1):74-81. [PubMed: 18977112]

Leal C, Chaix B. The influence of geographic life environments on cardiometabolic risk factors: systematic review, a methodological assessment and a research agenda. Obesity Reviews. 2010; 12(3):217-230. [PubMed: 20202135]

LeDoux TF, Vojnovic I. Going outside the neighborhood: the shopping patterns and adaptions of disadvantaged consumers living in the lower eastside neighborhoods of Detroit, Michigan. Health \& Place. 2013; 19:1-14. [PubMed: 23142639]

Mason M. Attributing activity space as risky and safe: the social dimension and meaning of place for urban adolescents. Health \& Place. 2010; 16:926-933. [PubMed: 20537934] 
Matthews S. The salience of neighborhood, some lessons from sociology. American Journal of Preventive Medicine. 2008; 34(4):257-259. [PubMed: 18312814]

Matthews S, Detwiler J, Burton L. Geo-ethnography: coupling geographic information analysis techniques with ethnographic methods in urban research. Cartographica. 2005; 40:75-90.

McGuirt J, Jilcott S, Vu M, Keyserling T. Conducting community audits to evaluate community resources for healthful life-style behaviors: an illustration from eastern North Carolina. Preventing Chronic Disease. Nov.2011 8(6) 2011.

Meegan R, Mitchell A. It's not community round here, it's neighborhood: neighborhood change and cohesion in urban regeneration policies. Urban Studies. 2001; 38(12):2167-2194.

Mitchell, A. The ESRI Guide to GIS Analysis. Vol. 2. ESRI Press; Redlands, CA: 2005.

Morland K, Wing S, Diez Roux A. The contextual effect of the local food environment on residents' diets: the atherosclerosis risk in communities study. American Journal of Public Health. 2002; 92(11):1761-1767. [PubMed: 12406805]

Morland K, Filomena S. Disparities in the availability of fruits and vegetables between racially segregated urban neighborhoods. Public Health Nutrition. 2007; 10(12):1481-1489. [PubMed: 17582241]

Nicotera N. Measuring neighborhood: a conundrum for human services researchers and practitioners. American Journal of Community Psychology. 2007; 40:26-51. [PubMed: 17577660]

Oliver L, Schuurman N, Hall A. Comparing circular and network buffers to examine the influence of land use on walking for leisure and errands. International Journal of Health Geographics. 2007; 6:41. doi:10.1186/1476-072X-6-41. [PubMed: 17883870]

Purcell M. Urban democracy and the Local Trap. Urban Studies. 2006; 43:1921-41.

Purcell M, Brown J. Against the Local Trap: scale and the study of environment and development. Progress in Development Studies. 2005; 5:279-297.

Rainham D, McDowell I, Krewski D, Sawada M. Conceptualizing the healthscape: contributions of time geography, location technologies and spatial ecology to place and health research. Social Science \& Medicine. 2010; 70:668-676. [PubMed: 19963310]

Raynor G. Conventional and ecological public health. Public Health. 2009; 123(9):587-591. [PubMed: 19748106]

Rundle A, Neckerman K, Freeman L, Lovasi G, Purciel M, Quinn J, Richards C, Sircar N, Weiss C. Neighborhood food environment and walkability predict obesity in New York City. Environmental Health Perspectives. 2009; 117(3):442-447. [PubMed: 19337520]

Saarloos D, Kim J, Timmerman H. The built environment and health: introducing individual spacetime behavior. International Journal of Environmental Research and Public Health. 2009; 6(6): 1724-1743. [PubMed: 19578457]

Sampson R, Morenoff J, Gannon-Rowley T. Assessing "neighborhood effects": social processes and new directions in research. Annual Review of Sociology. 28:443-478.

Scaglioni S, Salvioni M, Galimberti C. Influence of parental attitudes in the development of children eating behaviour. British Journal of Nutrition. 2008; 99(Supplement 1):S22-S25. [PubMed: 18257948]

Sharkey J, Johnson C, Dean W. Food access and perceptions of the community and household food environment as correlates of fruit and vegetable intake among rural seniors. BMC Geriatrics. 2010; 10:32. doi: 10.1186/1471-2318-10-32. [PubMed: 20525208]

Sherman J, Spencer J, Preisser J, Gesler W, Arcury T. A suite of methods for representing activity space in a healthcare accessibility study. International Journal of Health Geographics. 4:24. [PubMed: 16236174]

Siegel, S.; Castellan, J. Non-Parametric Statistics for the Behavioral Sciences. 2nd ed.. McGraw-Hill; New York: 1988.

Spielmann S, Yoo E. The spatial dimensions of neighborhood effects. Social Science \& Medicine. 2009; 68(6):1098-1105. [PubMed: 19167802]

Townley G, Kloos B, Wright P. Understanding the experience of place: expanding methods to conceptualize and measure community integration of persons with serious mental illness. Health \& Place. 2009; 15:52-531. 
Census, US. [Mar. 17, 2013] State and County Quick Facts. 2013. at: http://quickfacts.census.gov/qfd/ states/37/37147.html

USDA (U.S. Department of Agriculture). [Mar. 17, 2013] Rural-Urban Continuum Codes. 2013a. at: http://www.ers.usda.gov/data-products/rural-urban-continuum-codes.aspx

USDA (U.S. Department of Agriculture). [Mar. 17, 2013] Food Environment Atlas. 2013b. at: http:// www.ers.usda.gov/data-products/food-environment-atlas.aspx

Villaneuva K, Giles-Corti B, Bulsara M, McCormack G, Timperio A, Middleton N, Beesley B, Trapp G. How far do children travel from their homes? Exploring children's activity spaces in their neighborhood. Health \& Place. 2012; 18:263-272. [PubMed: 22001753]

Weiss L, Ompad D, Galeo S, Vlahov D. Defining neighborhood boundaries for urban health research. American Journal of Preventive Medicine. 2007; 6:S154-S159. [PubMed: 17543706]

Wild R, Taylor E, Knehans A, Cleaver V. Matriarchal model for cardiovascular prevention. Obstetrical \& Gynecological Survey. 1994; 49(2):147-52. [PubMed: 8164913]

Zenk S, Schulz A, Matthews S, Odoms-Young A, Wilbur J, Wegrzyn L, Gibbs K, Braunschweigh C, Stokes C. Activity space environment and dietary and physical activity behaviors: a pilot study. Health \& Place. 2011; 17:1150-1161. [PubMed: 21696995] 


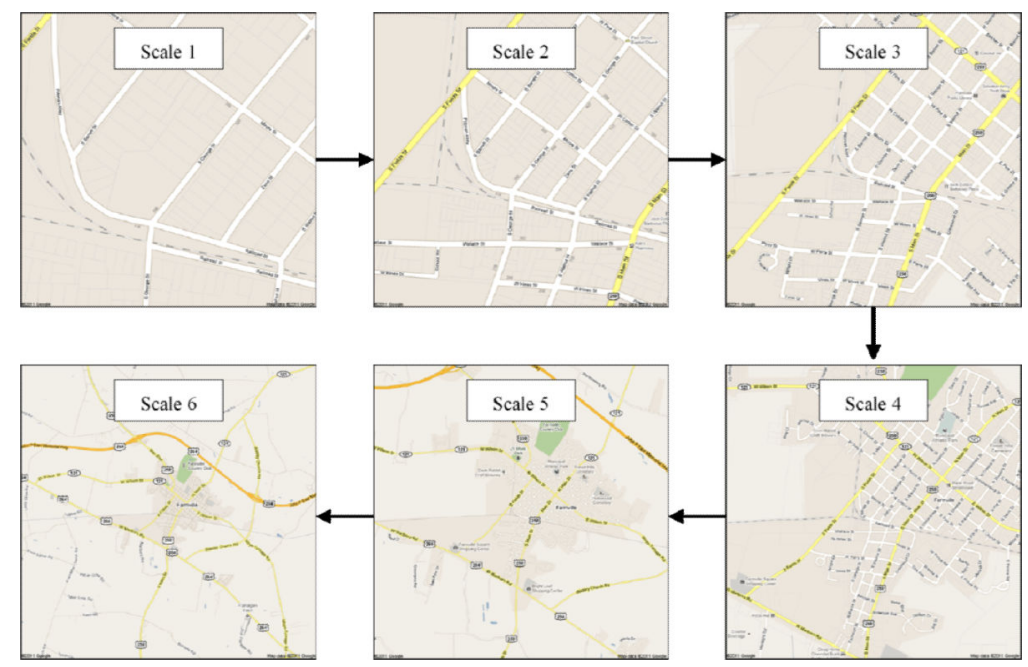

Fig. 1.

Blank sketch maps for a hypothetical participant at map scales: 1:2,000, 1:4,000; 1:8,000; $1: 16,000 ; 1: 32,000 ; 1: 64,000$. 


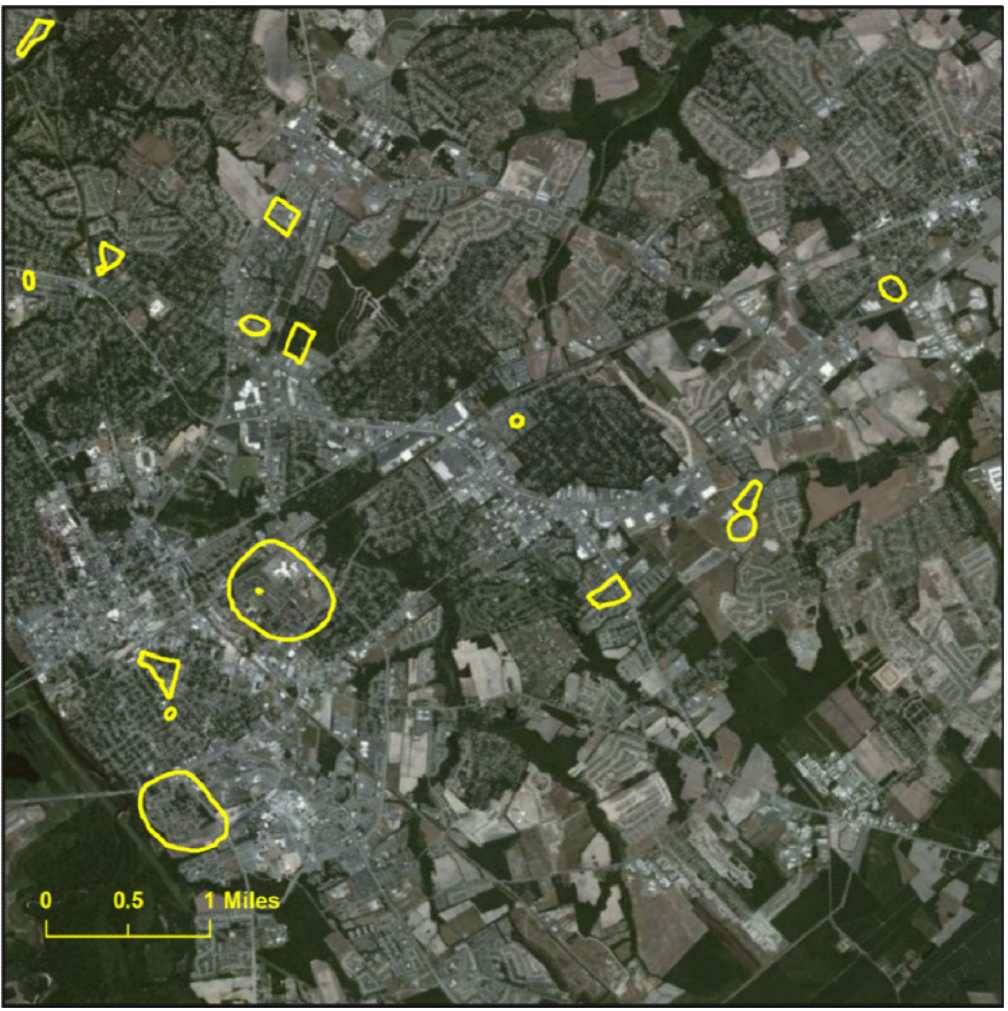

Fig. 2.

Self-defined sketch neighborhoods for selected participants, map image randomly rotated for confidentiality. 


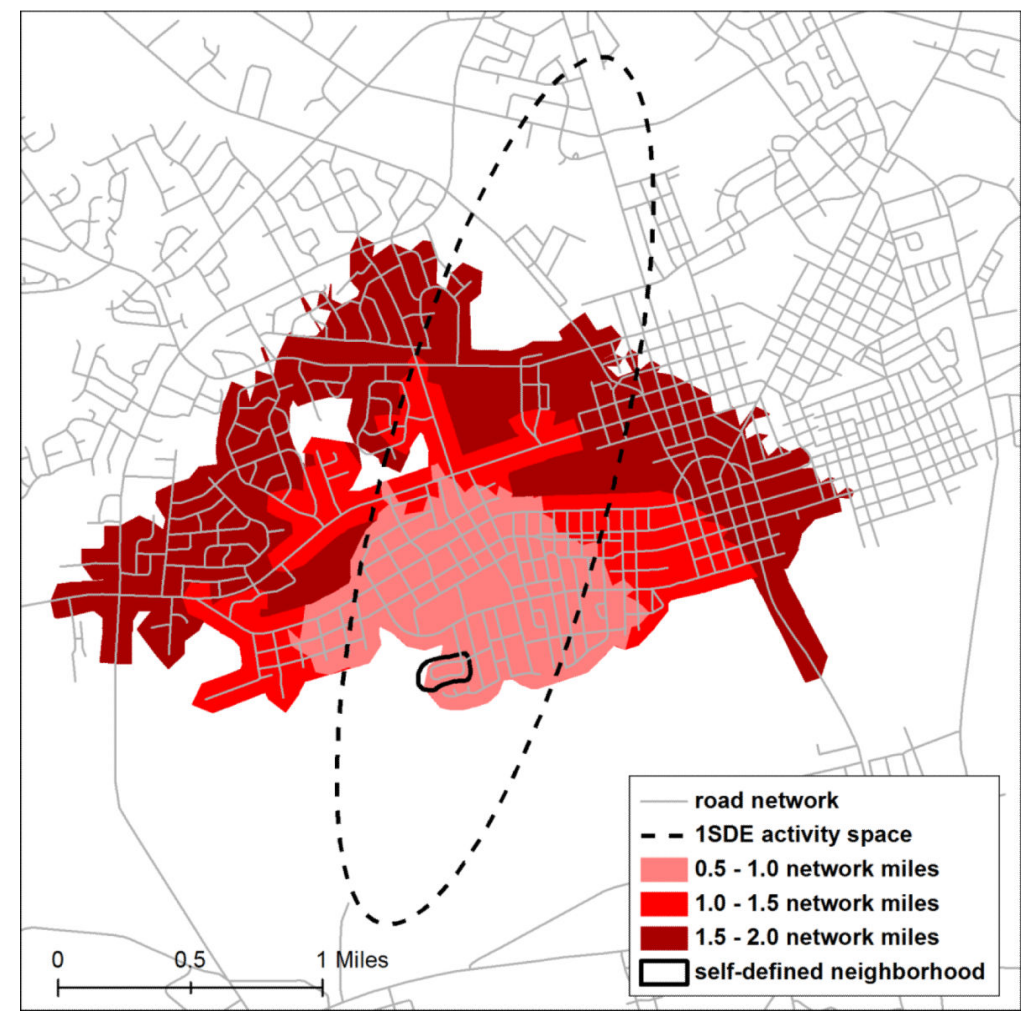

Fig. 3.

A participant's neighborhoods and activity space, map image randomly rotated for confidentiality. 


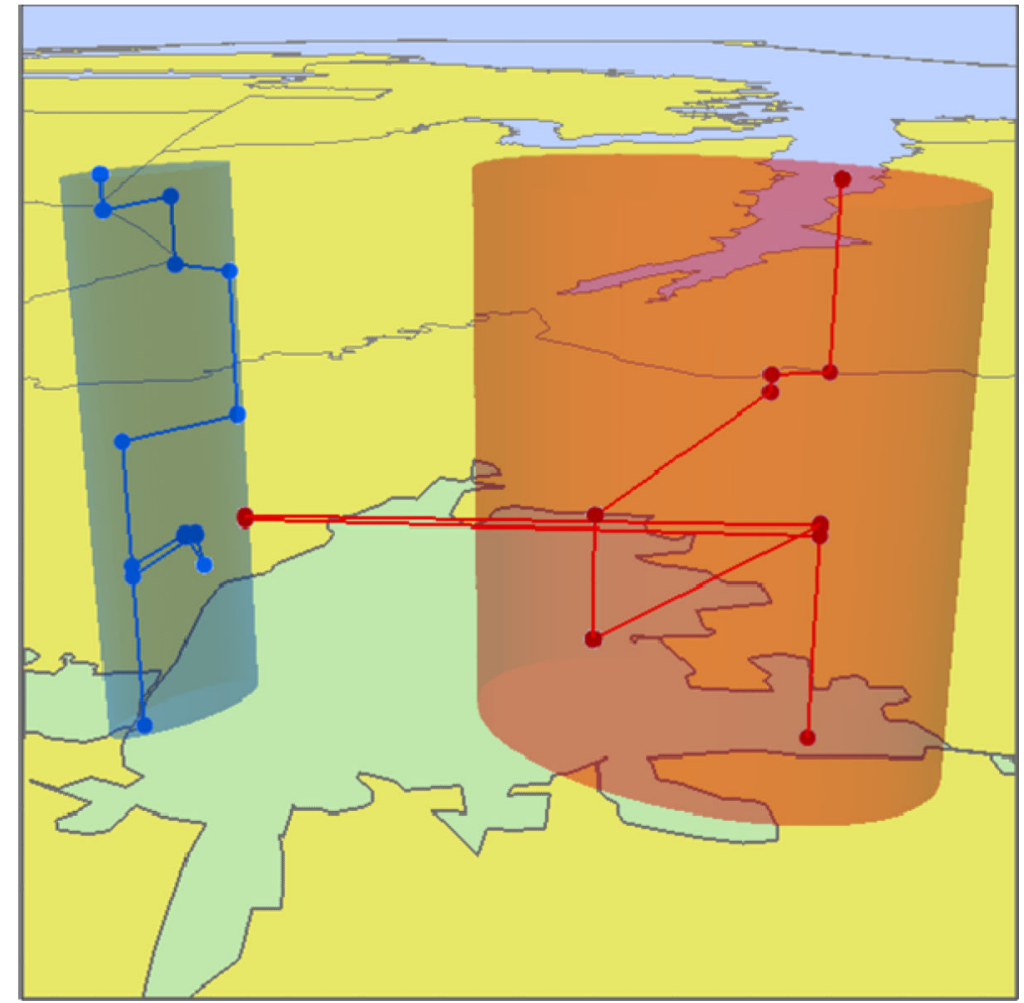

Fig.4.

Time-weighted, 1 standard deviational ellipses (SDE) and time-space paths for two selected participants. Vertical dimension represents a single 24-hour day. Paths are for one 24-hour day, ellipses are based on a 3-day period, green shaded and bounded area is city of Greenville. 


\section{Table 1}

Frequencies of map selected by participants to draw self-defined neighborhoods by cartographic scale.

\begin{tabular}{lrr}
\hline Cartographic scale & Count & $\%$ \\
$1: 2,000$ & 2 & 5.5 \\
$1: 4,000$ & 15 & 41.7 \\
$1: 8,000$ & 14 & 38.9 \\
$1: 16,000$ & 3 & 8.3 \\
$1: 32,000$ & 1 & 2.8 \\
$1: 64,000$ & 1 & 2.8 \\
\hline
\end{tabular}


Table 2

Descriptive statistics for neighborhoods and activity space area in square miles.

\begin{tabular}{lrr}
\hline Type of exposure area & Mean & Median \\
Self-defined neighborhood & 0.04 & 0.02 \\
Road Network neighborhood - 0.5 miles & 0.20 & 0.17 \\
Road Network neighborhood - 1.0 miles & 0.77 & 0.70 \\
Road Network neighborhood - 1.5 miles & 1.75 & 1.92 \\
Road Network neighborhood - 2.0 miles & 3.11 & 3.06 \\
Activity space - time weighted SDE & 26.36 & 8.72 \\
\hline
\end{tabular}

Friedman test: $\chi^{2}(5)=145.521, \mathrm{p} \leq 0.005$. 
Table 3

Pair-wise comparisons of area size and exposure for selected neighborhood types and activity space $(n=36)$.

\begin{tabular}{|c|c|c|c|}
\hline \multicolumn{4}{|l|}{ Area size } \\
\hline Sample 1 & Sample 2 & Test statistic & Adj. Sig. \\
\hline Self-defined nbh & Road Network nbh $1.0 \mathrm{mi}$ & -1.167 & 0.001 \\
\hline Self-defined nbh & Road Network nbh $2.0 \mathrm{mi}$ & -2.333 & 0.000 \\
\hline Self-defined nbh & Activity space, time weighted SDE & -2.278 & 0.000 \\
\hline Road Network nbh $1.0 \mathrm{mi}$ & Road Network nbh $2.0 \mathrm{mi}$ & -1.167 & 0.001 \\
\hline Road Network nbh $1.0 \mathrm{mi}$ & Activity space, time weighted SDE & -1.111 & 0.002 \\
\hline Road Network nbh $2.0 \mathrm{mi}$ & Activity space, time weighted SDE & -0.056 & 1.000 \\
\hline \multicolumn{4}{|l|}{ Supermarket exposure } \\
\hline Sample 1 & Sample 2 & Test Statistic & Adj. Sig. \\
\hline Self-defined nbh & Road Network nbh $1.0 \mathrm{mi}$ & -0.486 & 0.661 \\
\hline Self-defined nbh & Road Network nbh $2.0 \mathrm{mi}$ & -1.514 & 0.000 \\
\hline Self-defined nbh & Activity space, time weighted SDE & -1.667 & 0.000 \\
\hline Road Network nbh $1.0 \mathrm{mi}$ & Road Network nbh $2.0 \mathrm{mi}$ & -1.028 & 0.004 \\
\hline Road Network nbh $1.0 \mathrm{mi}$ & Activity space, time weighted SDE & -1.181 & 0.001 \\
\hline Road Network nbh $2.0 \mathrm{mi}$ & Activity space, time weighted SDE & -0.153 & 1.000 \\
\hline \multicolumn{4}{|l|}{ Farmers market exposure } \\
\hline Sample 1 & Sample 2 & Test Statistic & Adj. Sig. \\
\hline Self-defined nbh & Road Network nbh $1.0 \mathrm{mi}$ & -0.542 & 0.450 \\
\hline Self-defined nbh & Road Network nbh $2.0 \mathrm{mi}$ & -1.389 & 0.000 \\
\hline Self-defined nbh & Activity space, time weighted SDE & -1.736 & 0.000 \\
\hline Road Network nbh $1.0 \mathrm{mi}$ & Road Network nbh $2.0 \mathrm{mi}$ & -0.847 & 0.032 \\
\hline Road Network nbh $1.0 \mathrm{mi}$ & Activity space, time weighted SDE & -1.194 & 0.001 \\
\hline Road Network nbh $2.0 \mathrm{mi}$ & Activity space, time weighted SDE & -0.347 & 1.000 \\
\hline
\end{tabular}




\section{Table 4}

Supermarket and farmers' market exposure by type of exposure area $(n=36)$.

\begin{tabular}{|c|c|c|c|c|}
\hline \multirow[t]{2}{*}{ Type of Exposure Area } & \multicolumn{4}{|c|}{ Number of supermarkets } \\
\hline & 0 & 1 & 2 & $3+$ \\
\hline Self-defined neighborhood & 100.0 & 0.0 & 0.0 & 0.0 \\
\hline Road Network neighborhood -0.5 miles & 94.4 & 5.6 & 0.0 & 0.0 \\
\hline Road Network neighborhood - 1.0 miles & 61.1 & 36.1 & 2.8 & 0.0 \\
\hline Road Network neighborhood -1.5 miles & 36.1 & 27.8 & 33.3 & 2.8 \\
\hline Road Network neighborhood - 2.0 miles & 27.8 & 16.7 & 36.1 & 19.4 \\
\hline Activity space - time weighted SDE & 27.8 & 19.4 & 13.9 & 38.9 \\
\hline \multicolumn{5}{|l|}{ Friedman test: $\chi^{2}(5)=98.790, p \leq 0.005$} \\
\hline & \multicolumn{4}{|c|}{ Number of farmers markets } \\
\hline & 0 & 1 & 2 & $3+$ \\
\hline Self-defined neighborhood & 100.0 & 0.0 & 0.0 & 0.0 \\
\hline Road Network neighborhood -0.5 miles & 86.1 & 13.0 & 0.0 & 0.0 \\
\hline Road Network neighborhood - 1.0 miles & 63.9 & 36.1 & 0.0 & 0.0 \\
\hline Road Network neighborhood - 1.5 miles & 44.4 & 44.4 & 11.1 & 0.0 \\
\hline Road Network neighborhood - 2.0 miles & 30.6 & 44.4 & 25.0 & 0.0 \\
\hline Activity space - time weighted SDE & 22.2 & 33.3 & 19.4 & 25.0 \\
\hline Friedman test: $\chi^{2}(5)=94.453, \mathrm{p} \leq 0.005$ & & & & \\
\hline
\end{tabular}


Table 5

Mann-Whitney U test results comparing urban vs. rural and employed vs. unemployed $(n=34)$.

\begin{tabular}{|c|c|c|}
\hline Urban vs. Rural & & \\
\hline \multirow[t]{4}{*}{ Area } & Self-defined neighborhood & nd \\
\hline & Road network neighborhood - 1.0 miles & nd \\
\hline & Road network neighborhood - 2.0 miles & urban $>$ rural ${ }^{*}$ \\
\hline & Activity space - time weighted SDE & urban $<$ rural ${ }^{*}$ \\
\hline \multirow[t]{4}{*}{ Supermarket exposure } & Self-defined neighborhood & nd \\
\hline & Road network neighborhood - 1.0 miles & nd \\
\hline & Road network neighborhood - 2.0 miles & urban $>$ rural ${ }^{*}$ \\
\hline & Activity space - time weighted SDE & nd \\
\hline \multirow[t]{4}{*}{ Farmers' market exposure } & Self-defined neighborhood & nd \\
\hline & Road network neighborhood - 1.0 miles & nd \\
\hline & Road network neighborhood - 2.0 miles & urban $>$ rural $^{*}$ \\
\hline & Activity space - time weighted SDE & nd \\
\hline \multicolumn{3}{|l|}{ Employed vs. Unemployed } \\
\hline \multirow[t]{4}{*}{ Area } & Self-defined neighborhood & employed > unemployed ${ }^{*}$ \\
\hline & Road network neighborhood - 1.0 miles & nd \\
\hline & Road network neighborhood - 2.0 miles & nd \\
\hline & Activity space - time weighted SDE & nd \\
\hline \multirow[t]{4}{*}{ Supermarket exposure } & Self-defined neighborhood & nd \\
\hline & Road network neighborhood - 1.0 miles & nd \\
\hline & Road network neighborhood - 2.0 miles & nd \\
\hline & Activity space - time weighted SDE & nd \\
\hline \multirow[t]{4}{*}{ Farmers' market exposure } & Self-defined neighborhood & nd \\
\hline & Road network neighborhood - 1.0 miles & nd \\
\hline & Road network neighborhood - 2.0 miles & nd \\
\hline & Activity space - time weighted SDE & employed > unemployed ${ }^{*}$ \\
\hline
\end{tabular}

nd indicates no difference

*.

indicates $\mathrm{p} \leq 0.05$

Health Place. Author manuscript; available in PMC 2015 November 01. 